\title{
Hormonal Cycle Modulates Arousal Circuitry in Women Using Functional Magnetic Resonance Imaging
}

\author{
Jill M. Goldstein, ${ }^{1,2,3}$ Matthew Jerram, ${ }^{1,3}$ Russell Poldrack, ${ }^{4}$ Todd Ahern, ${ }^{2}$ David N. Kennedy, ${ }^{3,5}$ Larry J. Seidman, ${ }^{2,3}$ and \\ Nikos Makris ${ }^{3,5}$ \\ 'Department of Psychiatry, Harvard Medical School, Department of Psychiatry and Medicine, Brigham and Women's Hospital, Division of Women's \\ Health, Connors Center for Women's Health and Gender Biology, Boston, Massachusetts 02115, 2Department of Psychiatry at Beth Israel Deaconess \\ Hospital, Public Psychiatry Division at Massachusetts Mental Health Center and Massachusetts General Hospital, Boston, Massachusetts 02215, ${ }^{3}$ Athinoula \\ Martinos Center for Biomedical Imaging, Massachusetts General Hospital and Massachusetts Institute of Technology, Charlestown, Massachusetts 02129, \\ ${ }^{4}$ Department of Psychology, University of California at Los Angeles, Los Angeles, California 90095, and 5 Harvard Medical School, Departments of Neurology \\ and Radiology, Center for Morphometric Analysis, Massachusetts General Hospital, Charlestown, Massachusetts 02129
}

Sex-specific behaviors are in part based on hormonal regulation of brain physiology. This functional magnetic resonance imaging (fMRI) study demonstrated significant differences in activation of hypothalamic-pituitary-adrenal (HPA) circuitry in adult women with attenuation during ovulation and increased activation during early follicular phase. Twelve normal premenopausal women were scanned twice during the early follicular menstrual cycle phase compared with late follicular/midcycle, using negative valence/high arousal versus neutral visual stimuli, validated by concomitant electrodermal activity (EDA). Significantly greater magnitude of blood oxygenation level-dependent signal changes were found during early follicular compared with midcycle timing in central amygdala, paraventricular and ventromedial hypothalamic nuclei, hippocampus, orbitofrontal cortex (OFC), anterior cingulate gyrus (aCING), and peripeduncular nucleus of the brainstem, a network of regions implicated in the stress response. Arousal (EDA) correlated positively with brain activity in amygdala, OFC, and aCING during midcycle but not in early follicular, suggesting less cortical control of amygdala during early follicular, when arousal was increased. This is the first evidence suggesting that estrogen may likely attenuate arousal in women via cortical-subcortical control within HPA circuitry. Findings have important implications for normal sex-specific physiological functioning and may contribute to understanding higher rates of mood and anxiety disorders in women and differential sensitivity to trauma than men.

Key words: fMRI; emotion; arousal; hypothalamus; amygdala; women; HPA

\section{Introduction}

Knowledge of how gonadal and adrenal hormones regulate brain physiology provides a foundation for understanding sex-specific behaviors in health and disease. Here we argue that gonadal hormones regulate the affective arousal response through hypothalamic-pituitary-adrenal (HPA) circuitry. The menstrual cycle with its changing hormone levels provides an elegant natural paradigm in which to investigate this mechanism. This functional magnetic resonance imaging (fMRI) study demonstrated significant differences in the activation of arousal circuitry in adult women with attenuation during ovulation and increased activation during the early follicular menstrual cycle phase. Findings

\footnotetext{
Received June 2, 2005; revised Aug. 23, 2005; accepted Aug. 24, 2005.

This work was supported by National Institute of Mental Health Grant R01 MH56956 (J.M.G.) and an Athinoula Martinos Center for Biomedical Imaging at Massachusetts General Hospital Collaborative Start-Up Award. We thank Dr. Bernard Harlow for access to population sample, Drs. Stuart Tobet, Anne Klibanski, and Peter Schmidt for comments on previous drafts of this manuscript, and Valerie Thompson for manuscript preparation.

Correspondence should be addressed to Jill M. Goldstein, Connors Center for Women's Health and Gender Biol-

ogy, 1620 Tremont Street, Third Floor, Boston, MA 02120. E-mail: jill_goldstein@hms.harvard.edu.

T. Ahern's present address: Department of Neuroscience, Emory University, Atlanta, GA 30329.

DOI:10.1523/JNEUROSCI.2239-05.2005

Copyright $\odot 2005$ Society for Neuroscience $\quad 0270-6474 / 05 / 259309-08 \$ 15.00 / 0$
}

suggest that estrogen is likely attenuating arousal in women via cortical-subcortical control within the HPA circuitry.

Studies have identified brain regions involved in the stress response using aversive affective stimuli (Fuchs et al., 1985; Lang et al., 1997; Price, 1999), including central amygdala nuclei (cAMYG), ventromedial (VMN) and paraventricular (PVN) hypothalamic nuclei, hippocampus (HIPP), orbitofrontal cortex (OFC), anterior cingulate gyrus (aCING), and brainstem, including the peripeduncular nucleus (PPN) and locus ceruleus (Keverne, 1988; Price, 1999). These brain regions form a subcortical-cortical neural system within the HPA axis that regulates arousal, neuroendocrine, and affective responses (Fuchs et al., 1985; Swaab, 2004).

Previous imaging studies demonstrated activations of amygdala, OFC, aCING, hypothalamus, and insula using aversive stimuli (Simpson et al., 2000; Hariri et al., 2003; Taylor et al., 2003). Furthermore, normal women demonstrated greater responses to aversive arousal cues in amygdala, OFC, and aCING (Bradley et al., 2001; Wrase et al., 2003) and laterality differences (Schneider et al., 2000; Cahill et al., 2001; Canli et al., 2002) compared with men, likely associated with hormonal status (Fernandez et al., 2003). 
Positron emission tomography (PET) and functional magnetic resonance imaging studies of cognition, such as working memory or language, manipulated gonadal hormones in normal premenopausal and postmenopausal women and showed that estradiol and/or progesterone were significantly associated with differential activity in specific cortical regions (Berman et al., 1997; Shaywitz et al., 1999; Fernandez et al., 2003) and not cyclical gonadotropins (Berman et al., 1997). Behavioral and cognitive studies demonstrated variability dependent on menstrual cycle status (Sherwin, 1998; Shaywitz et al., 1999). Furthermore, some studies of the relationships between menstrual cycle phase and HPA activity demonstrated that progesterone enhanced HPA activity (Keller-Wood et al., 1988; Roca et al., 2003), whereas estrogen induced a blunted stress response (Lindheim et al., 1994).

Together, these studies suggest that gonadal hormones may significantly affect changes in brain activity associated with the stress response in women. However, no previous imaging studies have investigated the impact of hormonal variation in women on activation of the arousal circuitry. To investigate the potential impact of estrogen changes on brain activity, we compared women at early follicular (when estrogen and progesterone are relatively low) to midcycle timing (when progesterone remains relatively low but estrogen is high). We thus predicted, based in part on hormonal work by Keller-Wood et al. (1988), Roca et al. (2003), and Lindheim et al. (1994), significantly greater magnitude of brain activity in arousal circuitry during early follicular compared with midcycle timing in women.

\section{Materials and Methods}

Overview. Twelve normal premenopausal women underwent an fMRI paradigm of an aversive affective arousal task twice dependent on cycle timing. Visual image stimuli were based on an adapted version of the negative valence/high arousal and neutral valence/low arousal images from the International Affective Picture System (IAPS) (CSEA-NIMH, 1999), known to invoke the stress response system. Participants were brought in during the early follicular menstrual cycle phase (at onset of menstrual cycle) and during late follicular/midcycle periods. In a counterbalanced design, half of the participants were scanned within $2-3 \mathrm{~d}$ of onset of their menstrual cycle and returned for the second session $\sim 2$ weeks after their first visit. The other group was scanned $\sim 2$ weeks after their menses onset (i.e., during late follicular/midcycle timing) and returned $\sim 2$ weeks after that during menses onset. Studies have demonstrated lack of habituation in arousal response to IAPS images, thus validating the use of these images at two points in time (Bradley et al., 1996). Electrodermal activity (EDA) was simultaneously recorded during fMRI to validate physiologic arousal and examine associations with region-specific brain activity. Mood states were evaluated before and after scanning to control for state and trait-level anxiety and mood and to test for similarities across the two menstrual cycle phases.

Subjects. Participants in the present study were selected from a community sample drawn by the Harvard Mood and Cycles Study (Harlow et al., 1999). Women chosen were aged 36-40 years at the time of their entrance into the Harvard Mood and Cycles Study, right-handed, Caucasian, had at least high school education, English as their first language, no current psychiatric disorder, and free of Axis I psychiatric disorders during their lifetime [based on a systematic diagnostic interview (Harlow et al., 1999)]. The exclusion criteria also required absence of the following: (1) substance abuse within the last 6 months; (2) history of head injury with any documented cognitive sequelae or loss of consciousness greater than $5 \mathrm{~min}$; (3) neurologic disease; (4) mental retardation; and (5) medical illness that may significantly impair neurocognitive function. Furthermore, participants were required to have regular menstrual cycles for at least 1 year before inclusion in the present study. They knew their cycles well given that they had been followed in the Harvard study for $\sim 5$ years charting their menstrual cycles, validated by blood assays obtained regularly (Harlow et al., 1999).

Twelve right-handed, Caucasian women were systematically ascertained from this population for study. All participants were unmedicated and free of medical, neurological, and psychiatric illness and sensory impairments and had regular monthly cycles, at the least, over the past year. All gave informed consent and received an honorarium for participation. The mean age for the group was 43.9 years (SD of 1.45 years), and they ranged from 41 to 46 years old. The mean level of general intelligence was average (mean \pm SD IQ, $102.9 \pm 11.7$ ). All women were normally cycling confirmed by blood assays (estrogen, folliclestimulating hormone, luteinizing hormone, and progesterone) (Harlow et al., 1999). The time between their two visits was $\sim 2$ weeks.

Aversive arousal task. Stimulus materials were drawn from the IAPS (CSEA-NIMH, 1999), a well known set of images with demonstrated reliability and validity and known to invoke the stress response circuitry and behavior (Bradley et al., 1996; Lang et al., 1997). The IAPS is a system of color pictures previously rated by population samples along the dimensions of affective valence (pleasant- unpleasant) and arousal (calmaroused). Pictures were systematically drawn from the set according to affective valence (unpleasant and neutral) and arousal (high and low) based on normative ratings. Two sets of pictures, each containing 72 images, were selected: one of unpleasant valence and high arousal and the other of neutral valence and low arousal. To create the 72 fixation slides, the neutral valence/low arousal slides were transformed using Fourier transforms to create a set of slides with the same physical properties of the original but without content that was readily recognizable. These slides appear as extremely blurred versions of other slides with no recognizable content.

During the functional scans, participants were presented with three blocks of stimuli in a counterbalanced method. One block of stimuli consisted of fixation slides presented at the rate of one every $5 \mathrm{~s}$ for $30 \mathrm{~s}$. The other two blocks consisted of six IAPS pictures designated as arousing negative affect and six pictures designated as neutral in valence and low arousal. Six different pictures were presented for $5 \mathrm{~s}$ in each block, which lasted $30 \mathrm{~s}$. These blocks were repeated four times during each 6 min functional scan. Three functional scans were taken, resulting in 12 blocks of each condition. In all conditions, the participant was asked to press the button under her index finger each time the picture being presented changed to ensure attention to the presented stimuli. No cognitive task is required to respond to these images; thus, responses are unconfounded by cognitive capacities.

Imaging. Scanning was performed with a quadrature full head coil and a 1.5 T Sonata MR scanner (Siemens, Erlangen, Germany) modified for echo-planar imaging. A single slice two-dimensional spoiled gradient recall axial localizer scan was acquired, followed by a 60 slice sagittal localizer scan [conventional T1-weighted magnetization-prepared rapid-acquisition gradient echo (MPRAGE) gradient echo sequence; field of view (FOV), $23 \times 17.25 \mathrm{~cm}$; matrix, $256 \times 192$; in-plane resolution, $0.90 \mathrm{~mm}$; slice thickness, $2.8 \mathrm{~mm}$ ] to orient and, for subsequent scans, 15 contiguous axial slices along the anterior commissure-posterior commissure (AC-PC) line and covering the whole brain. This was followed by an automated shim procedure to improve $B_{0}$ field homogeneity and an MPRAGE T1-weighted flow-compensated scan (FOV, $40 \times$ $20 \mathrm{~cm}$; matrix, $512 \times 256$; in-plane resolution, $0.78 \mathrm{~mm}$; slice thickness, $7 \mathrm{~mm}$; 15 slices coplanar with the functional slices), which was used primarily to aid Talairach transformation during data analysis. The next scan was a 15-slice $\mathrm{T} 1$-weighted echo planar inversion recovery sequence [repetition time (TR), 20 s; echo time (TE), $40 \mathrm{~ms}$; inversion time, 1100 $\mathrm{ms}$; FOV , $40 \times 20 \mathrm{~cm}$; matrix, $256 \times 128$; in-plane resolution, $1.57 \mathrm{~mm}$; slice thickness, $7 \mathrm{~mm}$ ] coplanar to the functional images for anatomic localization. Finally, a series of functional scans were acquired, using an asymmetric spin echo, T2*-weighted sequence (TR, $2000 \mathrm{~ms}$; TE, $70 \mathrm{~ms}$; refocusing pulse offset by $-25 \mathrm{~ms}$; one excitation; FOV, $40 \times 20 \mathrm{~cm}$; matrix, $128 \times 64$; in-plane resolution, $3.125 \mathrm{~mm}$; slice thickness, $7 \mathrm{~mm}$; 15 contiguous slices along the AC-PC line). This pulse sequence has excellent sensitivity to parenchymal signal changes concurrent with experimental perturbation and reduced macrovascular sensitivity. Functional scans were acquired for 184 time points per experimental run (four 
time points of scanner shimming and 180 time points of A-B-A-B blocks). Three sets of functional scans were acquired. Functional images were visually examined in Statistical Parametric Mapping (SPM) to ensure whole-brain coverage. This inspection demonstrated coverage of regions of interest (ROIs) with the exception of the scans of two subjects who did not have adequate signal in the region of the hypothalamus and brainstem to perform additional analyses. These subjects were not included in the examination of brainstem and hypothalamus regions.

Psychophysiological measures. Physiological measures were measured continuously during functional imaging to validate arousal. EDA was recorded using sensors attached to the participant's fingers, expressed in microsiemens. These data were stored and analyzed on a Macintosh PowerPC (Apple Computers, Cupertino, CA) using PowerLab software (ADInstruments, Castle Hill, Australia). An average EDA level for each condition was calculated (negative valence/high arousal, neutral valence/ low arousal, and fixation) by averaging the EDA values for all of the blocks within each condition across the scans within one session. This led to separate values for the negative valence/high arousal, neutral valence/ low arousal, and fixation conditions for the early follicular and midcycle conditions. Difference scores were calculated as were average EDA values for the conditions for each participant.

Clinical assessments. Before and after each scan, participants were administered the Profile of Mood States (POMS) and the Spielberger StateTrait Anxiety Inventory (STAI) to control for mood state in analyses of brain activity and examine consistency across visits within the women. Participants were asked to rate their current mood. The POMS is an adjective checklist of 72 items. Each item is rated by the participant on a scale from 0 to 4 , with 0 indicating that the adjective does not apply at all and 4 indicating that the adjective describes the emotion state extremely well. Six scales are included in the POMS: anxiety, vigor, fatigue, confusion, anger, and depression. An overall score for mood state is derived as well. The value of each scale is determined by summing the number rating of each adjective in the scale and then dividing the sum by the total number of items in the scale. $\mathrm{T}$ scores for the main scale and for each subscale were computed for each subject to assess current mood before and after scanning.

The Spielberger State-Trait Anxiety Inventory is a questionnaire designed to assess an individual's perception of her current level of anxiety and her general level of anxiety. The individual rates 40 statements regarding feelings of anxiety on a scale from 1 to 4 , in which 1 indicates that the statement poorly reflects her feelings of anxiety and 4 indicates that the statement accurately reflects her feelings of anxiety. The first 20 statements reflect how the individual feels in general and reflect trait-level anxiety. The second 20 statements reflect current feelings of anxiety and reflect state-level anxiety. The ratings for the statements in each set of 20 are then summed to determine a total score for each set. The STAI was administered before and after scanning to assess state and trait-level anxiety and to test for similarities across the two menstrual cycle phases.

Participants also received the Vocabulary and Block Design subtests of the Wechsler Abbreviated Intelligence Scale (WASI), which were used as an estimate of current general intellectual function, and the Reading subtest of the Wide Range Achievement Test-Revised (WRAT-R), used as an estimate of intellectual potential to describe the sample of women. Age scaled scores were used for the WASI and WRAT-R variables. All of the interviews were performed by one of the authors (M.J.).

Experimental procedures. On arrival, the study was explained in detail, and informed consent was obtained. After giving consent, participants were administered the mood measures. After entry into the magnet room, participants were given the arousal task instructions and fit with earplugs to attenuate scanner noise. Participants lay prone on the scanner gurney, and foam padding was placed across the forehead to prevent head motion. Physiological monitoring sensors were placed on the fingers of the left hand of the participant, and the participant was given a response box with two buttons for the right hand. The head coil was then placed over the head, and the participant was placed into the scanner.

A Macintosh G4 Computer (Apple Computers) running MacStim experimental presentation program (WhiteAnt Occasional Publishing, West Melbourne, Australia) was used to produce the visual stimuli. Visual stimuli were presented via an liquid crystal display projector through a custom lens onto a screen situated in the magnet bore. These stimuli were viewed through a mirror attached to the head coil. The computer was in the control room outside the Faraday shield of the scanner. Stimuli were presented as described previously. When scanning was completed, the participant was again administered the POMS and state portion of the STAI. This procedure was repeated during the second visit.

Data analysis. fMRI data were preprocessed using SPM (Friston et al., 1995) and using custom routines in Matlab (MathWorks, Natick, MA). Preprocessing included correction for acquisition timing across slices, correction for bulk-head motion, and spatial smoothing with a Gaussian filter ( $8 \mathrm{~mm}$ at full-width half-maximum). No individual runs exhibited excessive head motion $(>5 \mathrm{~mm})$. After motion correction and spatial smoothing, images for each subject were spatially normalized using nonlinear volume-based spatial normalization techniques within SPM. The template used by SPM is the standard brain template developed at the Montreal Neurological Institute (MNI), and coordinates reported by SPM are convertible into standard Talairach space. After preprocessing, statistical analysis was performed at the single-subject level using SPM. SPM treats each voxel according to a general linear model, taking into account the intrinsic autocorrelation in fMRI data imposed by the slow hemodynamic response. Given that this was a block design, each epoch of trials was modeled using a boxcar function convolved with a canonical hemodynamic response function. Low-frequency components of the fMRI signal were modeled as confounding covariates using a set of cosine bias functions to increase sensitivity to signals of interest. Specific comparisons of interest were tested using linear contrasts, and SPM maps were created based on these contrasts. At the individual subject level, these contrasts represent the fixation condition (Fourier-transformed slides) from either the negatively valenced, high arousal condition or the neutral valenced, low arousal condition. Brain activations in response to the fixation slides were not analyzed separately. These contrast values (which represent estimates of the mean signal change at each voxel) were used in statistical analyses.

Results from the individual subject level were submitted to a secondlevel analysis in which subjects were treated as a random effect (i.e., for any particular effect, the variance used to test that effect was effect by subject variance). For a single group, activation was assessed using a one-sample $t$ test to compare the value of the contrast images against zero. For multiple groups, ANOVA was used to compare the size of a particular effect between groups.

ROI analyses were also performed. To define the ROIs functionally, the statistical map of the arousal condition by menstrual condition analysis was examined at the uncorrected $p<0.005$ level using a minimum size of four voxels for inclusion as a "significant" activation. Activated clusters within the ROIs were localized by visual inspection of our neuroanatomist (NM) and comparison to the neuroanatomical atlas. The NM was blind to study hypotheses and specific nuclei of interest before identification of the significant blood oxygen level-dependent (BOLD) signal intensity differences in activation during early follicular compared with midcycle, thus underscoring the validity of the neural network activated by our stimuli. The clusters were areas of signal change that varied with the task and were significantly different from the surrounding voxels as determined by SPM. After identifying clusters within the ROIs, it was possible to use SPM to overlay those clusters on the statistical maps of each individual. These template overlays allowed the acquisition of signal change values in the regions of interest for statistical comparison. Values indicated the degree of change in magnetic resonance signal detected between one of the experimental conditions and the fixation condition and were expressed in percentage signal change. To acquire a signal change value between the experimental conditions, the comparison-tofixation value of the neutral valence/low arousal condition was subtracted from the comparison-to-fixation value of the negative valence/ high arousal condition. Signal change values within each ROI were averaged. Effect sizes (ESs) presented in SD units represent differences between negative versus neutral signal intensity changes at two points in the menstrual cycle.

Finally, given that we had specific hypotheses about a set number of brain regions, we used the approach in SPM99 referred as small volume corrections (SVC). This process applies when one has a limited number 
of comparisons performed and uses functionally or anatomically defined limits. For these analyses, anatomically defined limits were used to test for activations within specific regions of interest, which included the amygdala, hypothalamus, hippocampus, brainstem, orbitofrontal cortex, anterior cingulate gyrus, and insula. Anatomic borders were defined using overlays created to match the borders of the hypothesized regions on the canonical brain provided with SPM99. Using the SVC tool in SPM99, activations within the borders of the overlays were identified, again using a minimum size of four voxels at the $p<0.005$ level of significance.

Each woman was used as her own control. Two groups were formed for some of the analyses: one of women during the late follicular/ midcycle peak phase and one of women in the early follicular stage. To examine the specificity of the findings regarding menstrual status, two other groups were formed: one of women during their first visit and one of women during their second (i.e., cycle timing was unaccounted for). Data analyzed included percentage signal intensity change values within the ROIs from fMRI scan, POMS T scores and difference scores, STAI scores and difference scores, and physiological measures. Results showed no significant differences in mood states across the two menstrual cycle phases.

\section{Results}

Tables 1-4 (below) show the significant activations comparing negative with neutral stimuli.

Table 1 shows the comparison of negative to neutral stimuli in women during the early follicular menstrual phase. As seen in Table 1, we demonstrated significant positive BOLD signal intensity changes from negative to neutral stimuli during the early follicular cycle phase in our brain regions of interest [i.e., cAMYG, PVN, PPN, OFC (BA47, BA11), and aCING (BA32); at a four voxel minimum, $p<.005$ (uncorrected)]. For the more stringent test (i.e., corrected for multiple comparisons at $p<0.005)$, the central amygdala and OFC (BA10) remained significant. In Table 2, during midcycle, significant positive BOLD changes in hypothesized regions were in CAMYG, PPN, and OFC (BA11), with CAMYG and OFC remaining significant at the corrected level.

For the main hypotheses in this study, most importantly, Tables 3 and 4 present the differences between BOLD signal intensity changes in negative versus neutral stimuli during early follicular compared with midcycle menstrual timing. State-trait measures of anxiety and mood rated before and after scanning at each session and assessed for the previous week were not significantly different, suggesting that women were in similar clinical states (at low normative levels) at both scanning sessions. When activations during the two
Table 1. Regions of activation (BOLD signal intensity differences) comparing aversive affective with neutral visual stimuli during the early follicular menstrual phase

\begin{tabular}{|c|c|c|c|c|c|c|c|}
\hline Region & Hemisphere & $x$ & $y$ & $Z$ & $Z$ & Voxels & SVC $p$ value \\
\hline \multicolumn{8}{|l|}{ Hypothesized regions } \\
\hline Amygdala & $\mathrm{L}$ & -24 & -9 & -12 & $4.01^{*}$ & 44 & 0.005 \\
\hline Amygdala & $\mathrm{R}$ & 24 & 0 & -21 & $3.71^{*}$ & 26 & 0.009 \\
\hline \multicolumn{8}{|l|}{ Hypothalamus } \\
\hline (PVN) & $\mathrm{L}$ & -3 & -9 & 3 & $2.67^{*}$ & 4 & 0.48 \\
\hline Brainstem (PPN) & $\mathrm{L}$ & -9 & -21 & -24 & $2.83^{*}$ & 6 & 0.12 \\
\hline BA10 & $M$ & 0 & 57 & -12 & $3.95^{*}$ & 60 & 0.006 \\
\hline BA10 & $\mathrm{R}$ & 15 & 66 & 18 & $3.19 *$ & 6 & 0.83 \\
\hline BA10 & $\mathrm{R}$ & 6 & 63 & 9 & $2.92^{*}$ & 12 & 0.40 \\
\hline BA11 & $\mathrm{R}$ & 27 & 30 & -18 & $3.45^{*}$ & 17 & 0.23 \\
\hline BA47 & $\mathrm{L}$ & -39 & 30 & -15 & $3.25^{*}$ & 23 & 0.19 \\
\hline BA32 & $\mathrm{L}$ & -12 & 48 & 9 & $3.41^{*}$ & 8 & 0.29 \\
\hline \multicolumn{8}{|l|}{ Other regions } \\
\hline BA17 & $\mathrm{R}$ & 15 & -78 & -3 & 6.31 & 7230 & \\
\hline BA45 & $\mathrm{R}$ & 57 & 33 & 12 & 4.94 & 24 & \\
\hline BA45 & $\mathrm{L}$ & -51 & 30 & 12 & 4.04 & 144 & \\
\hline BA20 & $\mathrm{L}$ & -36 & -6 & -42 & 3.97 & 16 & \\
\hline BA8 & $\mathrm{R}$ & 9 & 42 & 45 & 3.50 & 11 & \\
\hline BA21 & $\mathrm{R}$ & 60 & 0 & -21 & 3.44 & 13 & \\
\hline BA21 & $\mathrm{L}$ & -54 & -6 & -21 & 2.70 & 5 & \\
\hline BA7 & $\mathrm{R}$ & 27 & -57 & 57 & 3.33 & 48 & \\
\hline Pallidum & $\mathrm{L}$ & -9 & 0 & 0 & 3.20 & 7 & \\
\hline Thalamus & $\mathrm{L}$ & -6 & -15 & 3 & 3.19 & 6 & \\
\hline BA4 & $\mathrm{R}$ & 39 & -3 & 48 & 2.85 & 6 & \\
\hline BA9 & $\mathrm{L}$ & -3 & 60 & 27 & 2.74 & 14 & \\
\hline
\end{tabular}

Regions given in approximate Brodmann areas (BA); coordinates in MNI space listed $p$ levels for cluster-level comparison (functional activations identified by voxel-level comparison). L, Left; $R$, right; $M$, medial.

*Zscores are significant at $p<0.005$ with a minimum of four voxels activated.

Table 2. Regions of activation (BOLD signal intensity differences) comparing aversive affective with neutral visual stimuli during midcycle menstrual phase

\begin{tabular}{|c|c|c|c|c|c|c|c|}
\hline Region & Hemisphere & $x$ & $y$ & $Z$ & $Z$ & Voxels & SVC $p$ value \\
\hline \multicolumn{8}{|l|}{ Hypothesized regions } \\
\hline Amygdala & $\mathrm{L}$ & -21 & -3 & -21 & $4.15^{*}$ & 63 & 0.004 \\
\hline Brainstem (PPN) & $\mathrm{R}$ & 6 & -30 & -48 & $4.63^{*}$ & 25 & 0.67 \\
\hline BA11 & $\mathrm{R}$ & 6 & 18 & -9 & $3.83^{*}$ & 57 & 0.05 \\
\hline BA11 & $\mathrm{R}$ & 24 & 21 & -18 & $3.70^{*}$ & 31 & 0.12 \\
\hline \multicolumn{8}{|l|}{ Other regions } \\
\hline BA17 & $\mathrm{R}$ & 21 & -72 & 6 & 4.70 & 3354 & \\
\hline BA17 & $\mathrm{L}$ & -18 & -78 & 9 & 3.71 & 8 & \\
\hline BA18 & $\mathrm{L}$ & -15 & -93 & 21 & 3.18 & 11 & \\
\hline BA19 & $\mathrm{R}$ & 30 & -75 & 45 & 3.11 & 15 & \\
\hline BA45 & $\mathrm{L}$ & -57 & 24 & 12 & 3.73 & 20 & \\
\hline BA45 & $\mathrm{R}$ & 51 & 27 & 9 & 3.18 & 10 & \\
\hline BA6 & $\mathrm{R}$ & 39 & 0 & 54 & 3.39 & 21 & \\
\hline BA7 & $\mathrm{L}$ & -21 & -57 & 54 & 3.17 & 9 & \\
\hline BA7 & $\mathrm{R}$ & 30 & -54 & 57 & 3.05 & 16 & \\
\hline BA7 & $\mathrm{R}$ & 9 & -51 & 51 & 2.86 & 8 & \\
\hline BA20 & $\mathrm{R}$ & 45 & -6 & -39 & 2.99 & 6 & \\
\hline Putamen & $\mathrm{R}$ & 21 & -27 & -9 & 2.71 & 7 & \\
\hline BA21 & $L$ & -60 & -39 & -9 & 3.31 & 27 & \\
\hline
\end{tabular}

Regions given in approximate Brodmann areas (BA); coordinates in MNI space listed $p$ levels for cluster-level comparison (functional activations identified by voxel-level comparison). L, Left; $R$, right.

* $Z$ scores are significant at $p<0.005$ with a minimum of four voxels activated.

cycle conditions were directly compared, significant increases in BOLD signal changes in hypothesized cAMYG (ES, 3.38), VMN (ES, 3.36), OFC (BA47; ES, 3.03), aCING (BA32; ES, 2.80), and PPN (ES, 3.17) were found at early follicular compared with midcycle timing. Figures 1 and 2 illustrate the significant findings. Signal change values for early follicular, midcycle, and their differences for our regions of interest are presented in Table 4 and illustrated in Figure 3. 
Table 3. Regions of activation (BOLD signal intensity differences) between aversive affective to neutral visual stimuli during early follicular versus midcycle menstrual phases

\begin{tabular}{|c|c|c|c|c|c|c|c|}
\hline Region & Hemisphere & $x$ & $y$ & $Z$ & $Z$ & Voxels & SVC $p$ value \\
\hline \multicolumn{8}{|l|}{ Early follicular $>$ midcycle } \\
\hline \multicolumn{8}{|l|}{ Hypothesized regions } \\
\hline BA32 & $\mathrm{R}$ & 18 & 21 & 27 & $3.54^{*}$ & 9 & 0.30 \\
\hline Amygdala & $\mathrm{L}$ & -21 & -9 & -12 & $3.46^{*}$ & 18 & 0.02 \\
\hline Hypothalamus (PVN) & $\mathrm{L}$ & -6 & -6 & 3 & $3.36^{*}$ & 8 & 0.19 \\
\hline Hypothalamus (VMN) & $\mathrm{R}$ & 6 & 0 & -6 & $2.98^{*}$ & 4 & 0.03 \\
\hline Brainstem (PPN) & $\mathrm{R}$ & 12 & -27 & -6 & $3.17^{*}$ & 7 & 0.26 \\
\hline Hippocampus & $\mathrm{R}$ & 27 & -27 & -9 & $4.13^{*}$ & 6 & 0.29 \\
\hline BA47 & $\mathrm{R}$ & -30 & 39 & -6 & $3.45^{*}$ & 7 & 0.51 \\
\hline BA10 & $M / L$ & 0 & 54 & -9 & $3.24^{*}$ & 16 & 0.06 \\
\hline \multicolumn{8}{|l|}{ Other regions } \\
\hline Pallidum & $\mathrm{R}$ & 15 & -3 & -3 & 3.85 & 6 & \\
\hline BA18 & $\mathrm{L}$ & -18 & -81 & 0 & 3.92 & 48 & \\
\hline BA18 & $\mathrm{L}$ & -36 & -90 & 0 & 3.59 & 33 & \\
\hline BA18 & $\mathrm{L}$ & -21 & -96 & 9 & 3.43 & 11 & \\
\hline BA18 & $\mathrm{R}$ & 48 & -75 & -6 & 3.34 & 28 & \\
\hline BA18 & $\mathrm{R}$ & 21 & -84 & -15 & 3.03 & 22 & \\
\hline BA19 & $\mathrm{L}$ & -24 & -90 & 30 & 3.78 & 29 & \\
\hline BA37 & $\mathrm{L}$ & -60 & -60 & 0 & 3.60 & 71 & \\
\hline BA37 & $\mathrm{R}$ & 48 & -45 & -12 & 3.78 & 61 & \\
\hline BA30 & L & -9 & -45 & 3 & 3.36 & 5 & \\
\hline BA23 & $\mathrm{R}$ & 9 & -54 & 12 & 3.31 & 6 & \\
\hline BA39 & $\mathrm{L}$ & -39 & -72 & 27 & 3.30 & 18 & \\
\hline Cerebellum & L & -39 & -42 & -27 & 3.14 & 13 & \\
\hline \multicolumn{8}{|l|}{ Midcycle $>$ early follicular } \\
\hline \multicolumn{8}{|l|}{ All regions } \\
\hline BA40 & $\mathrm{R}$ & 54 & -42 & 36 & 3.43 & 14 & \\
\hline BA43 & $\mathrm{R}$ & 54 & -6 & 15 & 3.30 & 7 & \\
\hline
\end{tabular}

Regions given in approximate Brodmann areas (BA); coordinates in MNI space. L, Left; $R$, right; $M$, medial.

* $Z$ scores are significant at $p<0.005$ with a minimum of four voxels activated.

Table 4. Signal change values from neutral to negative conditions in early follicular and midcycle conditions in hypothesized regions

\begin{tabular}{|c|c|c|c|c|c|c|c|}
\hline Region & Hemisphere & $x$ & $y$ & $z$ & $\begin{array}{l}\text { Early } \\
\text { follicular }\end{array}$ & Midcycle & $\begin{array}{l}\text { Difference } \\
\text { between the } \\
\text { two conditions }\end{array}$ \\
\hline \multicolumn{8}{|l|}{ Hypothesized regions } \\
\hline aCING (BA32) & $\mathrm{R}$ & 18 & 21 & 27 & 0.01 & -0.13 & $0.14^{*}$ \\
\hline Amygdala & $\mathrm{L}$ & -21 & -9 & -12 & 0.23 & 0.07 & 0.16 \\
\hline Hypothalamus (PVN) & $\mathrm{R}$ & -6 & -6 & 3 & 0.19 & -0.03 & $0.22^{*}$ \\
\hline Hypothalamus (VMN) & $\mathrm{L}$ & 6 & 0 & -6 & 0.19 & -0.13 & 0.20 \\
\hline Brainstem (PPN) & $\mathrm{R}$ & 12 & -27 & -6 & 0.40 & 0.16 & $0.24^{*}$ \\
\hline Hippocampus & $\mathrm{R}$ & 27 & -27 & -9 & 0.28 & 0.12 & 0.16 \\
\hline OFC (BA47) & $R$ & -30 & 39 & -6 & 0.11 & -0.03 & 0.14 \\
\hline OFC (BA10) & M & 0 & 54 & -9 & 0.35 & 0.01 & $0.34^{* *}$ \\
\hline
\end{tabular}

Values derived from $8 \mathrm{~mm}$ sphere around center of activation (hypothalamus from $4 \mathrm{~mm}$ sphere) comparing early follicular $>$ midcycle phases. L, Left; $R$, right; $M$, medial.

${ }^{*} p<0.05 ;{ }^{* *} p<0.10$.

At the corrected level, central amygdala and hypothalamic VMN remained significant; medial OFC was at a $p<0.10$ level of significance (Table 3). However, even at the uncorrected level, we demonstrated that the majority of regions for which we showed significant differential activations dependent on cycle timing were those we hypothesized in the stress response network (Tables 3,4$)$. We thus demonstrated significant interaction effects of arousal by cycle timing in only 12 women scanned twice, reflecting the substantial effect sizes (i.e., $>3$ SD differences between activations in these regions at early follicular versus midcycle). [There were no significant activations in midcycle compared with early follicular timing in hypothesized regions, and only two regions were significant at the uncorrected level (in Table 3).]
To ensure discriminative validity, i.e., that BOLD signal differences were not simply attributable to differences in time itself, comparisons of visits 1 and 2 were evaluated (given that cycle timing was counterbalanced). Results showed no significant differences in brain activity in our regions of interest when cycle timing was unaccounted for.

Furthermore, to contribute to validation of the relationship between arousal and brain activity, EDA was recorded during each fMRI scanning session while observing the stimuli. At both cycle phases in women, EDA increased comparing neutrally valenced, low arousal with negatively valenced, high arousal stimuli, although arousal was higher during early follicular than late follicular/midcycle timing (mean increase, $0.10 \mu \mathrm{S}$ at early follicular and $0.05 \mu \mathrm{S}$ at midcycle). Previous functional imaging studies demonstrated inverse brain activity between the cortex and amygdala, which the authors argued reflected cortical control in the presence of arousal (Williams et al., 2004). In the present study during the early follicular phase, EDA negatively correlated with left cAMYG (Spearman's $r=-0.24$ ) and positively correlated with the cortex [right OFC $(r=0.31)$ and left aCING $(r=0.60)]$. In contrast during late follicular/midcycle timing, EDA positively correlated with left cAMYG (0.23) and the cortex [medial OFC (0.54), right OFC (0.26) and left aCING (0.59)]. Thus, the paradigm correlated with the expected physiological response modulated by cycle timing.

\section{Discussion}

Hypothalamic nuclei, such as PVN and VMN, in part regulate HPA hormones, connect to brainstem, including PPN, and are responsible for autonomic nervous system (ANS) function, adrenal and ovarian function and aggression, sexual and maternal behaviors, and the so-called stress response (Swaab, 2003, 2004). The hypothalamus integrates activity of the ANS and neuroendocrine system (Fuchs et al., 1985; Swaab, 2004). Central amygdala connects reciprocally with PVN, VMN, and PPN, modulates pituitary hormone secretion and reproductive functions (Zolovick, 1972), and is involved in aggression and the stress response. The amygdala and hypothalamus project to the brainstem, thus giving limbic system input to the control of the ANS (Armony and LeDoux, 2000). Medial/orbital prefrontal cortex provides frontal influence over autonomic and endocrine function (Price, 1999) and an integrative function between bodily states and goaldirected behavior. Shown in animal and human lesion studies, this arousal circuitry is also dense in sex steroid receptors, noradrenergic receptors (among other monoaminergic receptors), and oxytocin in some regions (Keverne, 1988; Feldman et al., 1995; 
Pacak et al., 1995; Tobet and Hanna, 1997; Price, 1999; Swaab, 2003, 2004).

The network of brain regions activated in our imaging study is consistent with previous studies of this aversive arousal circuitry in animals and humans and extend human imaging studies demonstrating for the first time activation of specific nuclei involved in the stress response. Furthermore, we demonstrated significant BOLD changes at two points in the menstrual cycle in these nuclei, suggesting hormonal effects on BOLD changes in brain regions associated with the stress response or HPA circuitry.

It is possible that either estrogen or progesterone may be contributing to the differences in brain activations within region during cycle changes. However, we would argue that it is estrogen or ratio of estrogen/androgens that have a moderating effect on a hyperaroused state in women. Women were scanned during early follicular (when estrogen and progesterone were low) and during late follicular/ midcycle peak (when estrogen was high and progesterone still low). Thus, this argues that, for sex steroid hormones, it is estrogen (or estrogen/androgen) driving the differences in activations rather than progesterone. Ovarian androgens follow cyclical patterns of estrogen but to a much lesser degree. Hormonal assays associated with signal intensity changes during scanning are needed for definitive validation of which hormone(s) account for the differences. However, our interpretation is consistent with a recent study demonstrating an inhibitory role of estradiol on neuronal activity in the mammillary body (Ishunina et al., 2000) and a study showing estradiol attenuating indices of noradrenergic function in menopausal women pre-estrogen versus post-estrogen implants (Best et al., 1992). It is also consistent with work demonstrating reduced free cortisol after stress challenge in women using estrogencontaining oral contraceptives (Kirschbaum et al., 1995, 1996), an effect of progesterone (and not estrogen) on enhanced HPA activity (Keller-Wood et al., 1988; Roca et al., 2003), and estradiol-induced blunting of the stress response (Lindheim et al., 1994). Furthermore, Taylor et al. (2000) theorized that the interaction of estrogen and oxytocin would lead to an attenuation of autonomic activity, such as our results indicated during midcycle when estrogen was high.

Furthermore, we would argue that it is the sex steroid hormones that are mediating the changes in brain activity more so than pituitary or gonadotropins, which also show cyclic changes. For example, a previous PET study with women on Lupron [a gonadotropin-releasing hormone $(\mathrm{GnRH})$ agonist] investigating
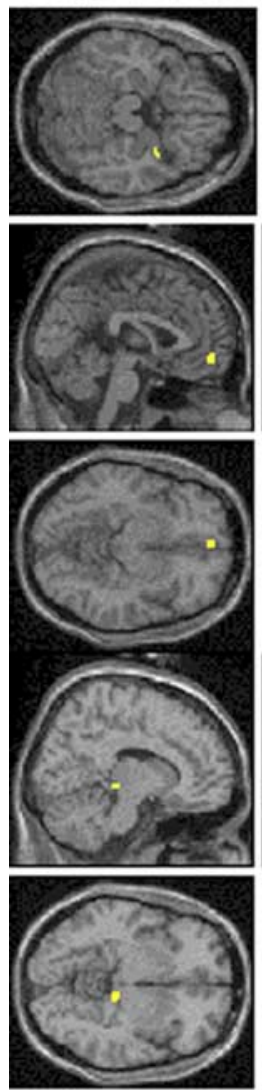
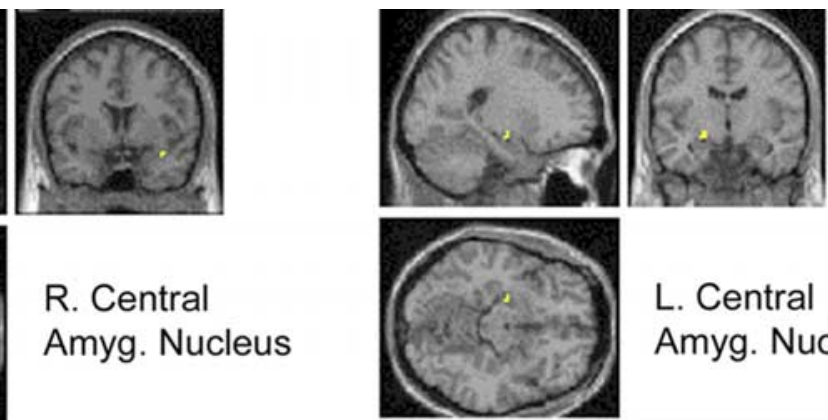

\section{Central Amyg. Nucleus}
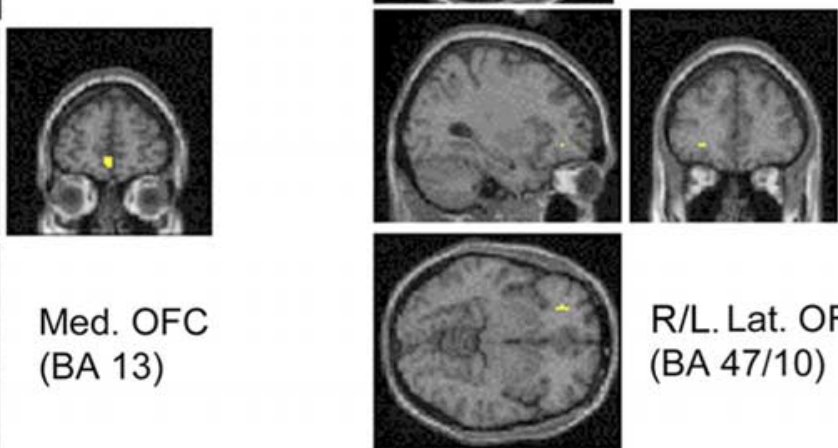

R/L. Lat. OFC (BA 47/10)
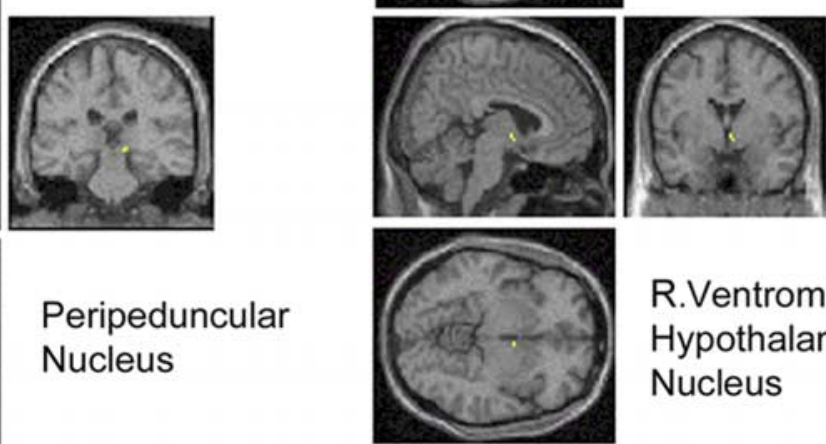

\section{R.Ventromedial Hypothalamic Nucleus}
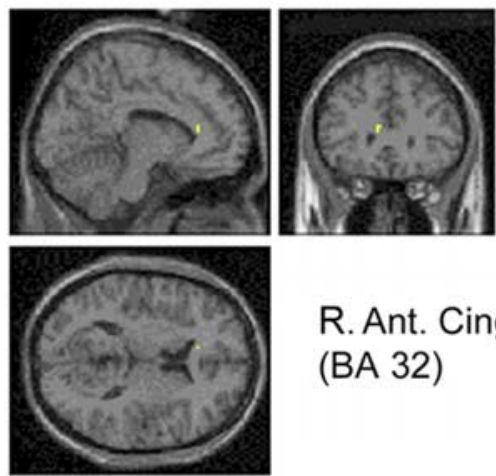

\section{R. Ant. Cingulate (BA 32)}

Figure 1. fMRI localization of significant differences in signal change within women comparing early follicular with midcycle menstrual phases during aversive arousal versus neutral visual stimuli. Images are derived from contrasts estimated by SPM of significant BOLD signal changes comparing aversive affective arousal stimuli versus neutral stimuli at early follicular versus midcycle menstrual timing, four-voxel minimum, controlled for multiple comparisons. R., Right; L., left; Amyg., amygdala; Med., medial; Lat., lateral; Ant., anterior.

working memory demonstrated that, when estrogen and progesterone were independently administered, prefrontal brain activity normalized (Berman et al., 1997), suggesting that the effect of a gonadotropin, $\mathrm{GnRH}$, on brain activity in prefrontal cortex was through its association with estrogen/progesterone regulation. Furthermore, previous fMRI working memory studies of postmenopausal women comparing on and off gonadal hormone replacement (Shaywitz et al., 1999) and other fMRI-gonadal hormone studies of language in premenopausal women (Fer- 


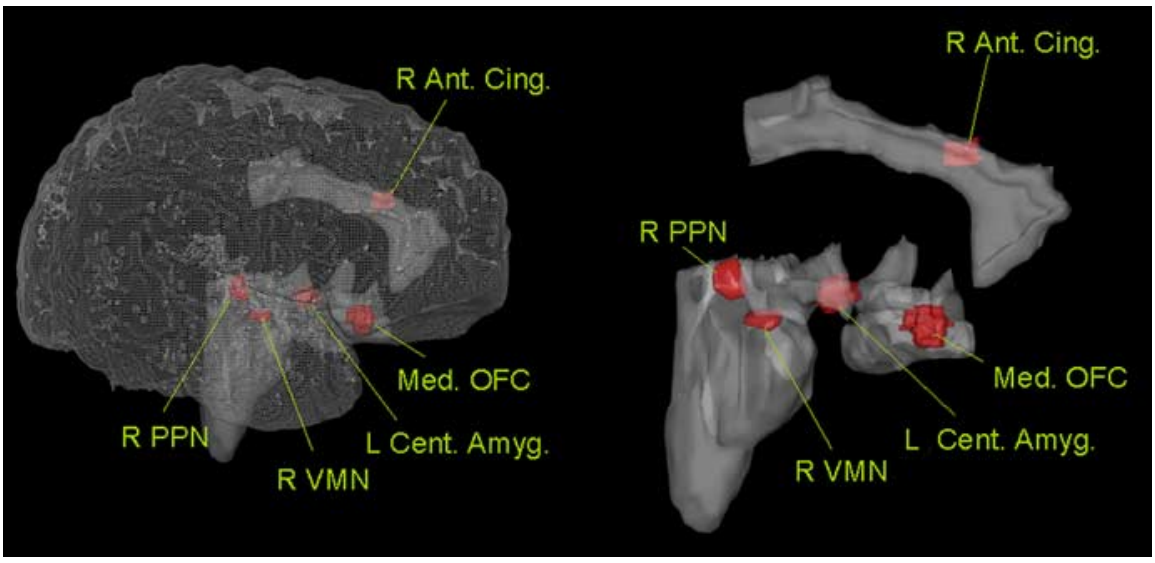

Figure 2. Three-dimensional images of anatomic localization of significant differences in signal change within women comparing early follicular with midcycle menstrual phases during aversive arousal versus neutral visual stimuli. Images represent the significant differences in BOLD signal intensity changes localized within anatomic regions (in red), corrected for multiple comparisons, comparing aversive affective stimuli versus neutral stimuli at two points in a woman's menstrual cycle (i.e., early follicular compared with midcycle menstrual phases). Regions of interest are shown within the mesh brain to illustrate the full context (i.e., whole brain) within which our brain regions of interest lie. L Cent. Amyg., Left central amygdala; Med. OFC, medial orbitofrontal cortex; R Ant. Cing., right anterior cingulate; RPPN, right peripeduncular nucleus; RVMN, right ventromedial hypothalamic nuclei.
1994). Furthermore, inhibiting aromatase during development, i.e., the enzyme that converts $\mathrm{T}$ to estradiol, resulted in a rise in $\mathrm{NE}$ in anterior frontal, insula, and cingulate cortices (Stewart and Rajabi, 1994), suggesting potentially a link between the arousal circuitry and gonadal hormones. Finally, animal evidence for the effect of estrogen on HPA hormones was seen when CRF-injected intracerebrally inhibited lordosis in ovariectomized estrogenprimed female rats (Sirinathsinghii et al., 1983), and the behavioral profile of lactating rats (when estradiol is high) showed reduced fear (Hansen et al., 1985). These animal and human studies during development and adulthood suggest that circulating estrogen in adulthood may have a regulatory effect on HPA hormones and NE released in frontal cortex, such that estrogen attenuates NE release and behaviors associated with corticotropin-releasing hormones and the stress response.

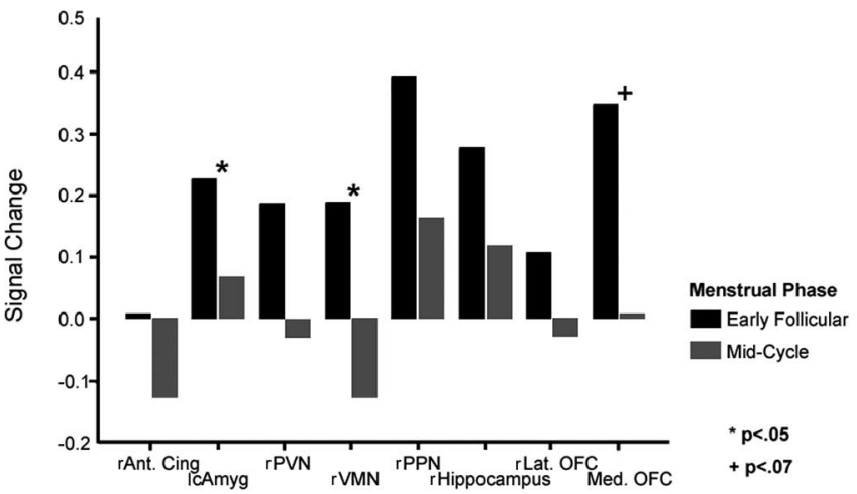

Figure 3. Signal intensity changes in BOLD activations comparing aversive affective with neutral stimuli in regions of interest at early follicular and late follicular/midcycle peak menstrual phases. Values indicate the degree of change in magnetic resonance signal detected between experimental condition and fixation and reported as percentage signal change. Comparison-to-fixation value of neutral valence/low arousal condition was subtracted from comparison-to-fixation value of negative valence/high arousal condition. Signal change values within each region of interest were averaged. Effect sizes presented in SD units represent differences between negative versus neutral signal intensity changes at early follicular and midcycle conditions. r, Right; Ant. Cing, anterior cingulate; IcAmyg, left central amygdala; Lat., lateral; Med., medial.

nandez et al., 2003) also demonstrated significant BOLD changes in cortical activity dependent on circulating gonadal hormone variations.

In fact, estrogen receptors $\alpha(\mathrm{ER} \alpha)$ and $\beta(\mathrm{ER} \beta)$ are located in cAMYG, HIPP, VMN, PVN, PPN, and cortex (Keverne, 1988; Ostlund et al., 2003; Bao et al., 2005). Although previous work demonstrated excitatory effects of $\operatorname{ER} \alpha, \operatorname{ER} \beta$ has shown nonexcitatory effects, thus suggesting that, through these receptors, estrogen may modulate autonomic and mood-related functions in relation to corticotropin-releasing factor (CRF) and norepinephrine (NE) release (Ostlund et al., 2003; Bao et al., 2005; Lund et al., 2005). In fact, a recent postmortem study demonstrated colocalization of CRH neurons with estrogen receptors in the PVN (Bao et al., 2005). Previous studies in animals demonstrated that NE levels during prenatal development were regulated by testosterone ( $\mathrm{T}$ ) and differentially by sex (Stewart and Rajabi,
We also demonstrated in our brain regions of interest significant relationships with arousal (measured by EDA) and the amygdala, which reversed directions depending on whether the woman was in early follicular or midcycle, suggesting a change in cortical/subcortical relationship dependent on cycle timing. That is, when arousal was high during late follicular/midcycle timing (i.e., when estrogen was high and progesterone low), arousal correlated positively with brain activity in the central amygdala and positively with OFC and the anterior cingulate, supporting previous work suggesting cortical control of arousal (Williams et al., 2004). However, during early follicular (when levels of estrogen and progesterone were low), there was a disconnection between arousal (operationalized with EDA) and amygdala activity, reflected in a negative correlation between the two, although the correlation of EDA with cortical activity remained positive. This suggests less control of amygdala activity by the cortex during the early follicular period, a period of increased arousal. Thus, the neural network through which ovulatory hormones exert their effect on lowering arousal may be in part through their effect on the relationships between amygdala with OFC and anterior cingulate gyrus, brain regions known to have relatively high densities of gonadal hormone receptors (Ostlund et al., 2003).

Consistent with this, we demonstrated in this study in women an attenuation of brain activity in nuclei that define the stress response in the presence of higher levels of estrogen (i.e., midcycle vs early follicular differences). These findings identify a neural pathway through which gonadal hormones may regulate arousal in women via cortical control of subcortical regions in HPA circuitry. In a previous study, we demonstrated normal sexual dimorphisms in these brain regions (Goldstein et al., 2001). Together with the functional imaging presented here, results have important implications for understanding sex-specific physiological functioning. These findings can be investigated in relation to the known higher rates of anxiety disorders, differential sensitivity to trauma in women compared with men, and development of potential sex-specific pharmacologic interventions targeted to specific nuclei, estrogen receptor activity, and/or noradrenergic function in these nuclei. Furthermore, our findings make sense from an evolutionary point of view, in that an 
attenuation of hyperarousal during the ovulatory period may increase availability, receptivity, and desire for procreation.

\section{References}

Armony JL, LeDoux J (2000) How danger is encoded: toward a systems, cellular, and computational understanding of cognitive-emotional interactions in fear. In: The new cognitive neurosciences, Ed 2 (Gazzaniga MS, ed), pp 1067-1079. Cambridge, MA: MIT.

Bao AM, Hestiantoro A, Van Someren EJ, Swaab DF, Zhou JN (2005) Colocalization of corticotropin-releasing hormone and oestrogen receptor- $\alpha$ in the paraventricular nucleus of the hypothalamus in mood disorders. Brain 128:1301-1313.

Berman KF, Schmidt PJ, Rubinow DR, Danaceau MA, Van Horn JD, Esposito G, Ostrem JL, Weinberger DR (1997) Modulation of cognition-specific cortical activity by gonadal steriods: a positron-emission tomography study in women. Proc Natl Acad Sci USA 94:8836-8841.

Best N, Rees MP, Barlow DH, Cowen PJ (1992) Effect of estradiol implant on noradrenergic function and mood in menopausal subjects. Psychoneuroendocrinology 17:87-93.

Bradley MM, Cuthbert BN, Lang PJ (1996) Picture media and emotion: effects of a sustained affective context. Psychophysiology 33:662-670.

Bradley MM, Cogispoti M, Sabatinelli D, Lang PJ (2001) Emotion and motivation. II. Sex differences in picture processing. Emotion 1:300-319.

Cahill L, Haier RJ, White NS, Fallon J, Kilpatrick L, Lawrence C, Potkin SG, Alkire MT (2001) Sex-related difference in amygdala activity during emotionally influenced memory storage. Neurobiol Learn Mem 75:1-9.

Canli T, Desmond JE, Zhao Z, Gabrieli JDE (2002) Sex differences in the neural basis of emotional memories. Proc Natl Acad Sci USA 99:10789-10794.

CSEA-NIMH (1999) International affective picture system: digitized photographs. Gainesville, FL: Center for Research in Psychophysiology, University of Florida.

Feldman S, Conforti N, Weidenfeld J (1995) Limbic pathways and hypothalamic neurotransmitters mediating adrenocortical responses to neural stimuli. Neurosci Biobehav Rev 19:235-240.

Fernandez G, Weis S, Stoffel-Wagner B, Tendolkar I, Reuber M, Beyenburg S, Klaver P, Fell J, de Greiff A, Ruhlmann J, Reul J, Elger CE (2003) Menstrual cycle-dependent neural plasticity in the adult human brain is hormone, task, and region specific. J Neurosci 23:3790-3795.

Friston KJ, Holmes KJ, Worsley JB, Poline JB, Frith C, Frackowiak RS (1995) Statistical parametric maps in functional imaging: a general linear approach. Hum Brain Mapp 2:189-210.

Fuchs SA, Edinger HM, Siegel A (1985) The organization of the hypothalamic pathways mediating affective defense behavior in the cat. Brain Res 330:77-92.

Goldstein JM, Seidman LJ, Horton NJ, Makris M, Kennedy DN, Caviness VS, Faraone SV, Tsuang MT (2001) Normal sexual dimorphism of the adult human brain assessed by in-vivo magnetic resonance imaging. Cereb Cortex 11:490-497.

Hansen S, Ferreira A, Selart ME (1985) Behavioural similarities between mother rats and benzodiazepine-treated non-maternal animals. Psychopharmacology (Berl) 86:344-347.

Hariri AR, Mattay VS, Tessitore A, Fera F, Weinberger DR (2003) Neocortical modulation of the amygdala response to fearful stimuli. Biol Psychiatry 53:494-501.

Harlow B, Cohen LS, Otto MW, Spiegelman D, Cramer DW (1999) Prevalence and predictors of depressive symptoms in older premenopausal women: the Harvard Study of Moods and Cycles. Arch Gen Psychiatry 56:418-424.

Ishunina T, Kruijver FP, Balesar R, Swaab DF (2000) Differential expression of estrogen receptor alpha and beta immunoreactivity in the human supraoptic nucleus in relation to sex and aging. J Clin Endocrinol Metab 85:3283-3291.

Keller-Wood M, Silbiger J, Wood CE (1988) Progesterone attenuates the inhibition of adrenocorticotropin responses by cortisol in nonpregnant ewes. Endocrinology 123:647-651.

Keverne EB (1988) Central mechanisms underlying the neural and neuroendocrine determinants of maternal behaviour. Psychoneuroendocrinology 13:127-141.

Kirschbaum C, Pirke KM, Hellhammer DH (1995) Preliminary evidence for reduced cortisol responsivity to psychological stress in women using oral contraceptive medication. Psychoneuroendocrinology 20:509-514.

Kirschbaum C, Platte P, Pirke KM, Hellhammer DH (1996) Adrenocortical activation following stressful exercise: further evidence for attenuated free cortisol responses in women using oral contraceptives. Stress Med 12:137-143.

Lang PJ, Bradley MM, Cuthbert MM (1997) Motivated attention: affect, activation, and action. In: Attention and orienting: sensory and motivational processes (Lang PJ, Simons RF, Balaban MT, eds), pp 97-135. Hillsdale, NJ: Erlbaum.

Lindheim SR, Legro RS, Morris RS, Wong IL, Tran DQ, Vijod MA, Stanczyk FZ, Lobo RA (1994) The effect of progestins on behavioral stress responses in postmenopausal women. J Soc Gynecol Investig 1:79-83.

Lund TD, Rovis T, Chung WC, Handa RJ (2005) Novel actions of estrogen receptor-beta on anxiety-related behaviors. Endocrinology 146:797-807.

Ostlund H, Keller E, Hurd YL (2003) Estrogen receptor gene expression in relation to neuropsychiatric disorders. Ann NY Acad Sci 1007:54-63.

Pacak K, Palkovits M, Kopin IJ, Goldstein DS (1995) Stress-induced norepinephrine release in the hypothalamic paraventricular nucleus and pituitary-adrenocortical and sympathoadrenal activity: in vivo microdialysis studies. Front Neuroendocrinol 16:89-150.

Price JL (1999) Prefrontal cortical networks related to visceral function and mood. Ann NY Acad Sci 877:383-396.

Roca CA, Schmidt PJ, Altemus M, Deuster P, Danaceau MA, Putnam K, Rubinow DR (2003) Differential menstrual cycle regulation of hypothalamic-pituitary-adrenal axis in women with premenstrual syndrome and controls. J Clin Endocrinol Metab 88:3057-3063.

Schneider F, Habel U, Kessler C, Salloum JB, Posse S (2000) Gender differences in regional cerebral activity during sadness. Hum Brain Mapp 9:226-238.

Shaywitz SE, Bennett A, Pugh KR, Fulbright RK, Skudlarksi P, Mencl WE, Constable RT, Naftolin F, Palter SF, Marchione KE, Katz L, Shankweiler DP, Fletcher JM, Lacadie C, Keltz M, Gore JC (1999) Effect of estrogen on brain activation patterns in postmenopausal women during working memory tasks. JAMA 281:1197-1202.

Sherwin BA (1998) Estrogen and cognitive functioning in women. Proc Soc Exp Biol Med 217:17-22.

Simpson JR, Öngür D, Akbudak E, Conturo TE, Ollinger JM, Snyder AZ, Gusnard DA, Raichle ME (2000) The emotional modulation of cognitive processing: an fMRI study. J Cogn Neurosci 12 [Suppl 2]:157-170.

Sirinathsinghji DJ, Rees LH, Rivier J, Vale W (1983) Corticotropinreleasing factor is a potent inhibitor of sexual receptivity in the female rat. Nature 305:232-235.

Stewart J, Rajabi H (1994) Estradiol derived from testosterone in prenatal life affects the development of catecholamine systems in the frontal cortex in the male rat. Brain Res 646:157-160.

Swaab DF (2003) The human hypothalamus. Basic and clinical aspects, Pt I, Nuclear of the hypothalamus. In: Handbook of clinical neurology, pp 476. Amsterdam: Elsevier.

Swaab DF (2004) The human hypothalamus. Basic and clinical aspects, Pt II, Neuropathology of the hypothalamus and adjacent brain structures. In: Handbook of clinical neurology, pp 596. Amsterdam: Elsevier.

Taylor SE, Klein LC, Lewis BP, Gruenewald TL, Gurung RA, Updegraff JA (2000) Biobehavioral responses to stress in females: tend-and-befriend, not fight-or-flight. Psychol Rev 107:411-429.

Taylor SF, Phan KL, Decker LR, Liberzon I (2003) Subjective rating of emotionally salient stimuli modulates neural activity. NeuroImage 18:650-659.

Tobet SA, Hanna IK (1997) Ontogeny of sex differences in the mammalian hypothalamus and preoptic area. Cell Mol Neurobiol 17:565-601.

Williams LM, Das P, Harris AW, Liddell BB, Brammer MJ, Olivieri G, Skerrett D, Phillips ML, David AS, Peduto A, Gordon E (2004) Dysregulation of arousal and amygdala-prefrontal systems in paranoid schizophrenia. Am J Psychiatry 161:480-489.

Wrase J, Klein S, Gruesser SM, Hermann D, Flor H, Mann K, Braus DF, Heinz A (2003) Gender differences in the processing of standardized emotional visual stimuli in humans: a functional magnetic resonance imaging study. Neurosci Lett 348:41-45.

Zolovick AJ (1972) Effects of lesions and electrical stimulation of the amygdala on hypothalamic-hypophyseal regulation. In: The neurobiology of the amygdala (Eleftheriou BE, ed), pp 745-762. New York: Plenum. 